\title{
Supporting Information for \\ Phase-Pure Wurtzite GaAs Nanowires \\ Grown by Self-Catalyzed Selective Area Molecular Beam Epitaxy for Advanced Laser Devices and Quantum Disks
}

Marvin M. Jansen 1,3,*, Pujitha Perla ${ }^{1,3}$, Mane Kaladzhian ${ }^{1,3}$, Nils von den Driesch ${ }^{1,3}$, Johanna Janßen ${ }^{1,3}$, Martina Luysberg ${ }^{3,4}$, Mihail I. Lepsa ${ }^{2,3}$, Detlev Grützmacher ${ }^{1,2,3}$ and Alexander Pawlis ${ }^{1,3, \#}$

1) Peter-Grünberg-Institute (PGI-9), Forschungszentrum Jülich GmbH, 52425 Jülich, Germany

2) Peter-Grünberg-Institute (PGI-10), Forschungszentrum Jülich GmbH, 52425 Jülich, Germany

3) JARA-Fundamentals of Future Information Technology, Jülich-Aachen Research Alliance, 52062 Aachen, Germany

4) Ernst Ruska-Centre for Microscopy and Spectroscopy with Electrons (ER-C-1), Forschungszentrum Jülich $\mathrm{GmbH}, 52425$ Jülich, Germany

\section{Corresponding Author}

Marvin Marco Jansen *E-Mail: m.jansen@fz-juelich.de

Alexander Pawlis \# E-Mail: a.pawlis@fz-juelich.de

\section{Table of Materials}

S1 Substrate overview and cooldown process .............................................................. 1

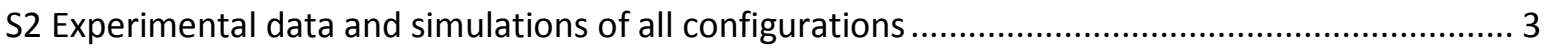

S3 Calculation and calibration of the material fluxes......................................................... 10

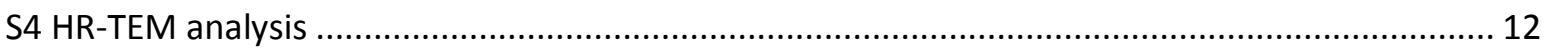

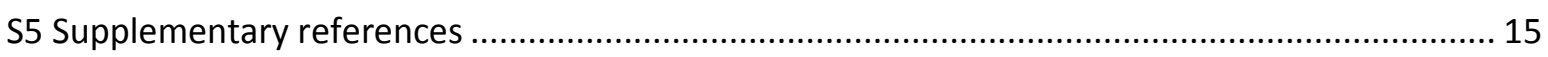




\section{Substrate overview}

Figure S1a depicts a SEM image of the hole array pattern (D80/P2) and the inset shows the hole morphology measured by AFM. Figure S1b is an overview SEM image of a NW array grown in config. A ( 45 min growth time). For the latter we obtained vertical NW yields up to nearly $70 \%$. The insert shows a small subset of the as-grown NWs each with a Ga droplet on top.

\section{Finished hole pattern}

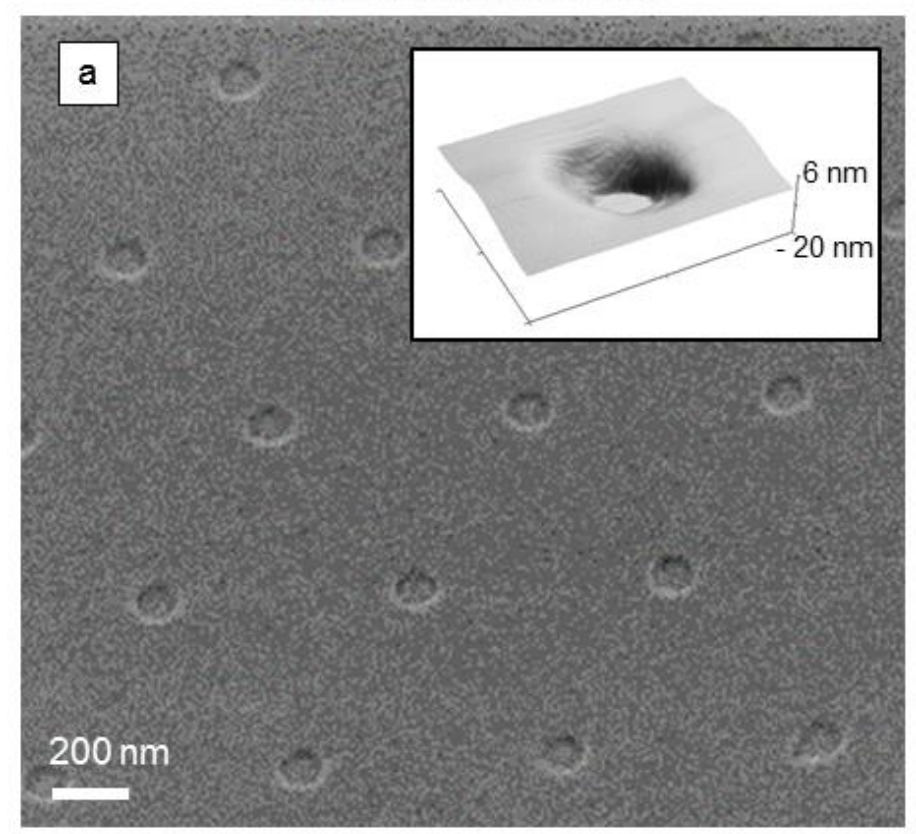

NW array

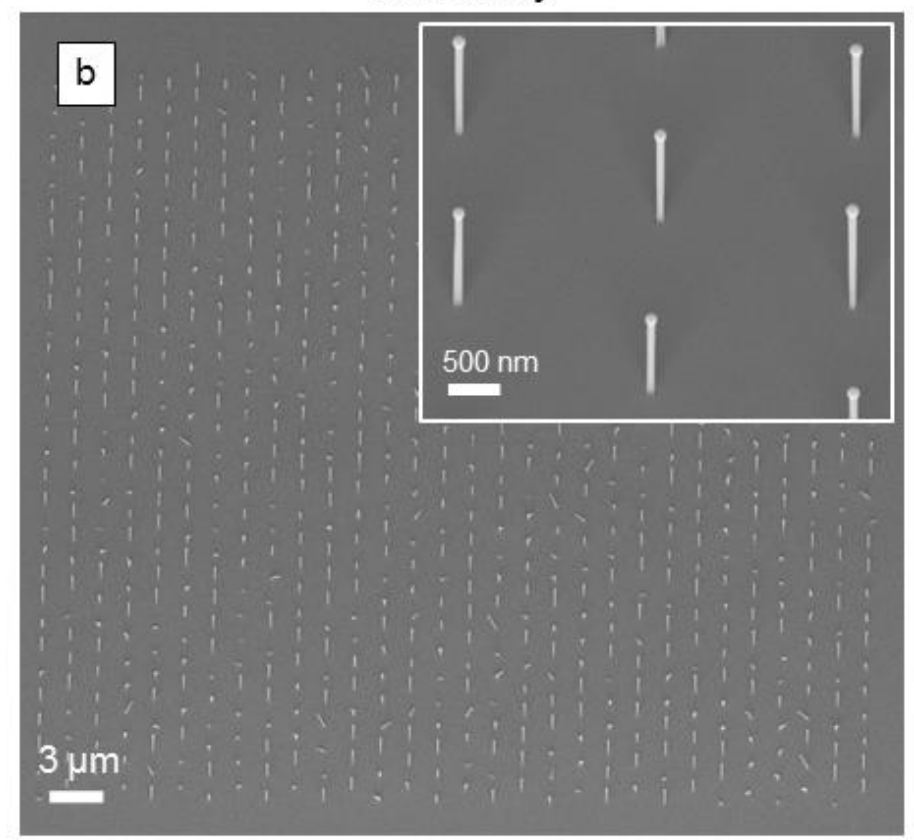

Figure S1. a) SEM image of the hole array pattern used for the D80/P0.5 configuration Inset: Topography of a single hole measured by AFM. b) Exemplary SEM images of the NW array in the D80/P2 configuration (config. A). Vertical oriented NW yields up to $70 \%$ are achieved. 


\section{Cooldown procedure}

After NW growth, all cell shutters including the main substrate shutter are closed immediately. The substrate temperature was then rapidly decreased to approx. $230{ }^{\circ} \mathrm{C}$ within $10 \mathrm{~min}$ to minimize the post-growth during the sample cooldown and to preserve the Ga droplet contact angle. A similar cooldown process and similar post-growth examination of the contact angle was also performed by Vettori et al. ${ }^{s 1}$ and represents the current technological standard.

Nevertheless, we cannot completely suppress post-growth of the NWs. Therefore, we examined the Ga droplet consumption during the cooldown process. After a regular NW growth run the remaining $\mathrm{As}_{4}$ BEP inside the MBE chamber is about $1 / 20$ of the BEP used for NW growth ( $>45 \mathrm{~min}$ ). It is worth mentioning that this value is substantially smaller $(\sim 1 / 100)$ for short growth times $(5-22.5 \mathrm{~min})$. Under these conditions an additional vertical NW growth of $100-200 \mathrm{~nm}$ was quantified by SEM and TEM analysis (e.g., see SEM analysis in Fig. S2) and an overall contact angle change from $125^{\circ}-130^{\circ}$ to $95^{\circ}-105^{\circ}$ was observed. Additionally, we performed a second growth run in which the Ga shutter was closed, while both, $A s_{4}$ and substrate shutter remained open for $10 \mathrm{~min}$ at the growth temperature. A similar experiment was conducted by Rieger et al.. ${ }^{s 2}$

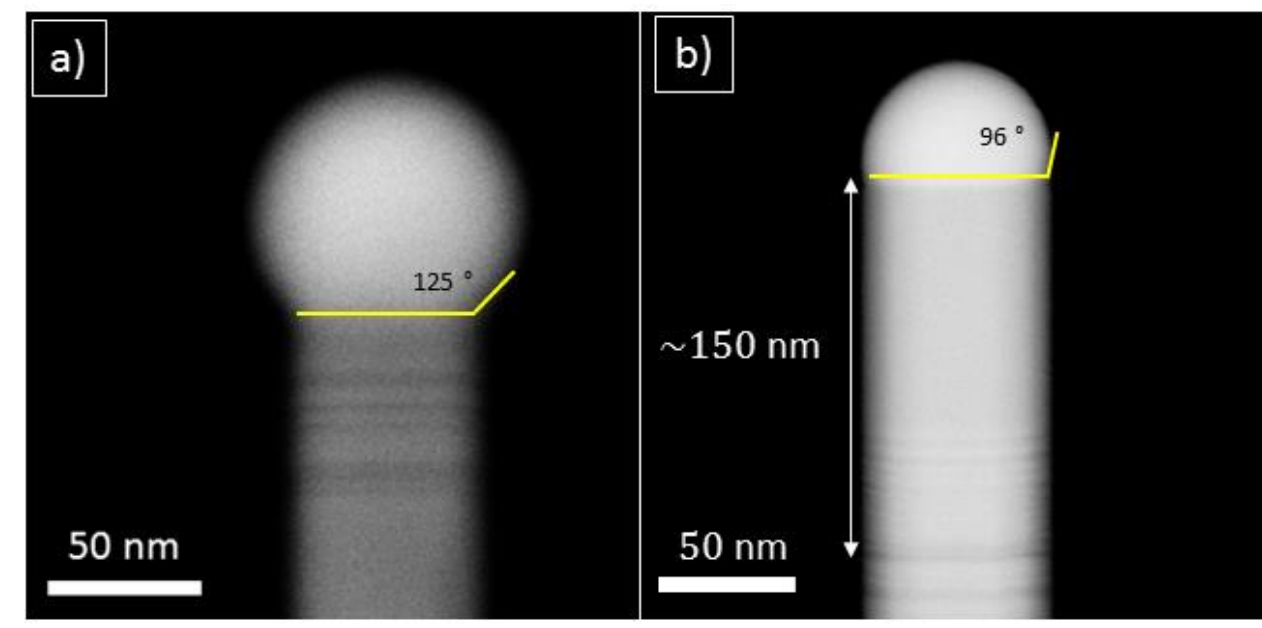

Figure S2. (a) Side view SEM image of a regular grown NW. (b) SEM image of a NW after 10 min $\mathrm{As}_{4}$ purge at the end of the NW growth.

Since the post-growth BEP of $\mathrm{As}_{4}$ is about $1 / 20-1 / 100$ of the BEP used for the regular growth, we estimate the regular post-growth with all shutters closed to about $10-20 \mathrm{~nm}$ and a reduction of the contact angle by less than $3^{\circ}$. Note, that the resulting contact angle variation due to post-growth is smaller than the error bar of $\pm 3-5^{\circ}$ considered in the experimental data and our model. 


\section{S2 Experimental data and simulations of all configurations}

\section{Overview of all configurations}

Fig. S3 shows an exemplary overview of SEM images taken for all configurations of each sample. The pitches are $0.5 \mu \mathrm{m}$ (P0.5), $1.0 \mu \mathrm{m}$ (P1.0), $2.0 \mu \mathrm{m}$ (P2.0) and $4 \mu \mathrm{m}$ (P4). For clarity we defined three subsets depending on the hole diameters found in our pre-patterned (PP) substrates: Subset $A$ has a hole diameter of $d_{A}=80 \mathrm{~nm}$ while subset B and subset $\mathrm{C}$ correspond to hole diameters of, $d_{B}=$ $60 \mathrm{~nm}$ and $d_{C}=40 \mathrm{~nm}$, respectively.

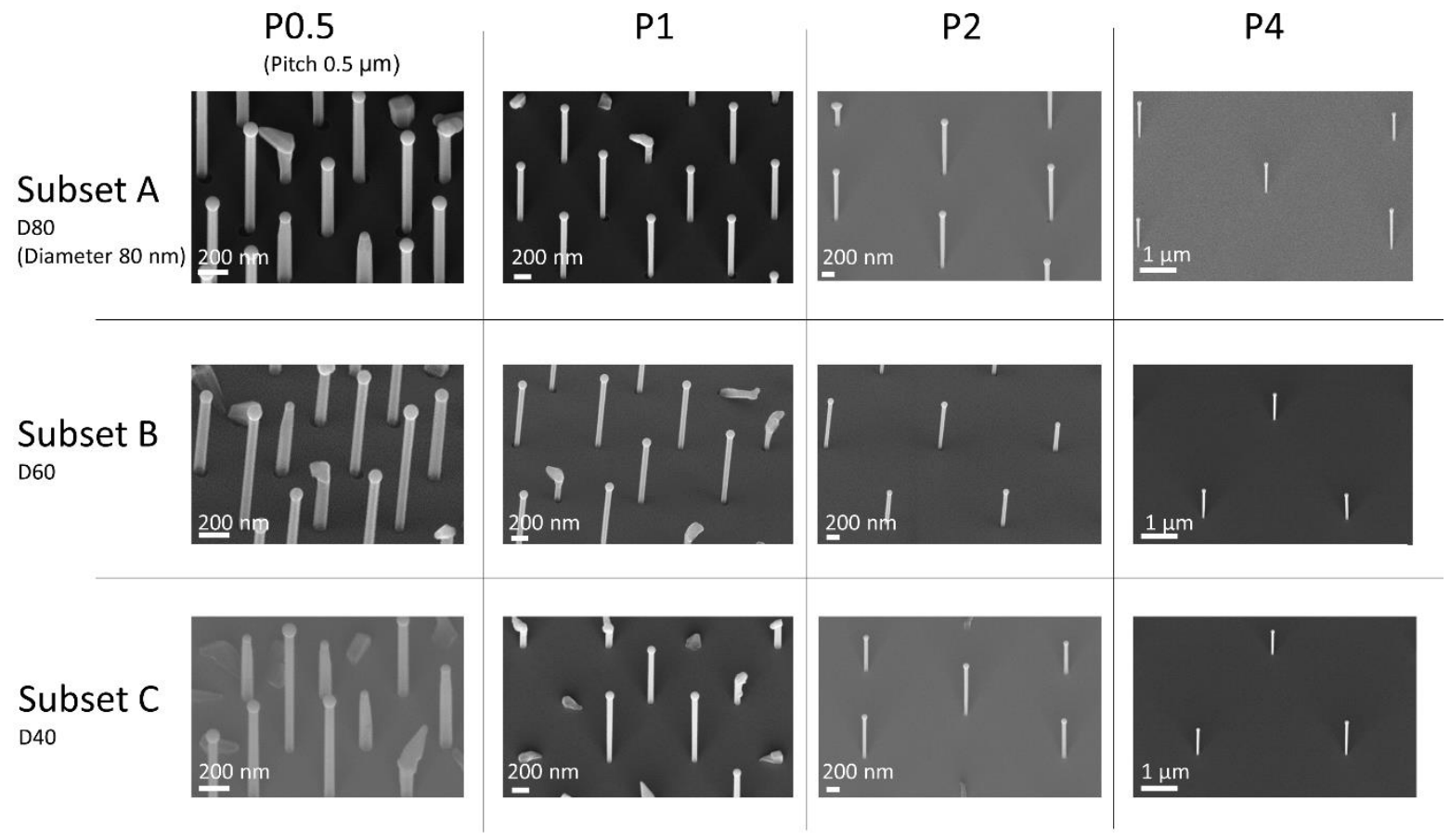

Figure S3. Overview SEM images of all analyzed configurations. The latter are divided into three subsets (A-C) distinguished by the hole diameter. The pitch is varied between $0.5 \mu \mathrm{m}$ and $4 \mu \mathrm{m}$ and the NWs growth time was $45 \mathrm{~min}$.

\section{Diameter and length evolution}

The pitch of the hole array was varied to investigate additional effects on the NW growth such as shadowing effects, re-emission from the oxide and nearest-neighbor interactions. We systematically observed and analyzed the main parameters of the NW growth as a function of the pitch for all investigated hole diameters. Our study revealed that the time evolution of NW length and diameter is similar for all pitches. At the beginning of NW growth (a few hundred nanometers of NW length), the share of $\mathrm{Ga}$ atoms impinging on the $\mathrm{SiO}_{2}$ and diffusing from the $\mathrm{SiO}_{2}$ to the Ga droplet is considerably high. If the pitch is in the order of the diffusion length of $\mathrm{Ga}$ on $\mathrm{SiO}_{2}$, a competitive collection of the total amount of $\mathrm{Ga}$ adatoms on the shared area between neighboring NWs might occur. Correspondingly, we investigated the NW diameter evolution as a function of the NW length for all configurations. 
Figs. S4 - S6 show the NW diameter as a function of the NW length obtained for all three subsets A-C. For each data point 5 - $15 \mathrm{NWs}$ were analyzed and the error bars reflect the standard deviation $\pm \sigma$ of the mean average values. We applied linear regressions for all configurations. The anisotropy factor $\gamma=d d_{N W} / d L$ and the initial NW diameter $d_{N W}(0)$ are included in Figs. S4 - S6. The linear fits are in good agreement with the experimental dataset of all pitch and diameter configurations.

Subset A

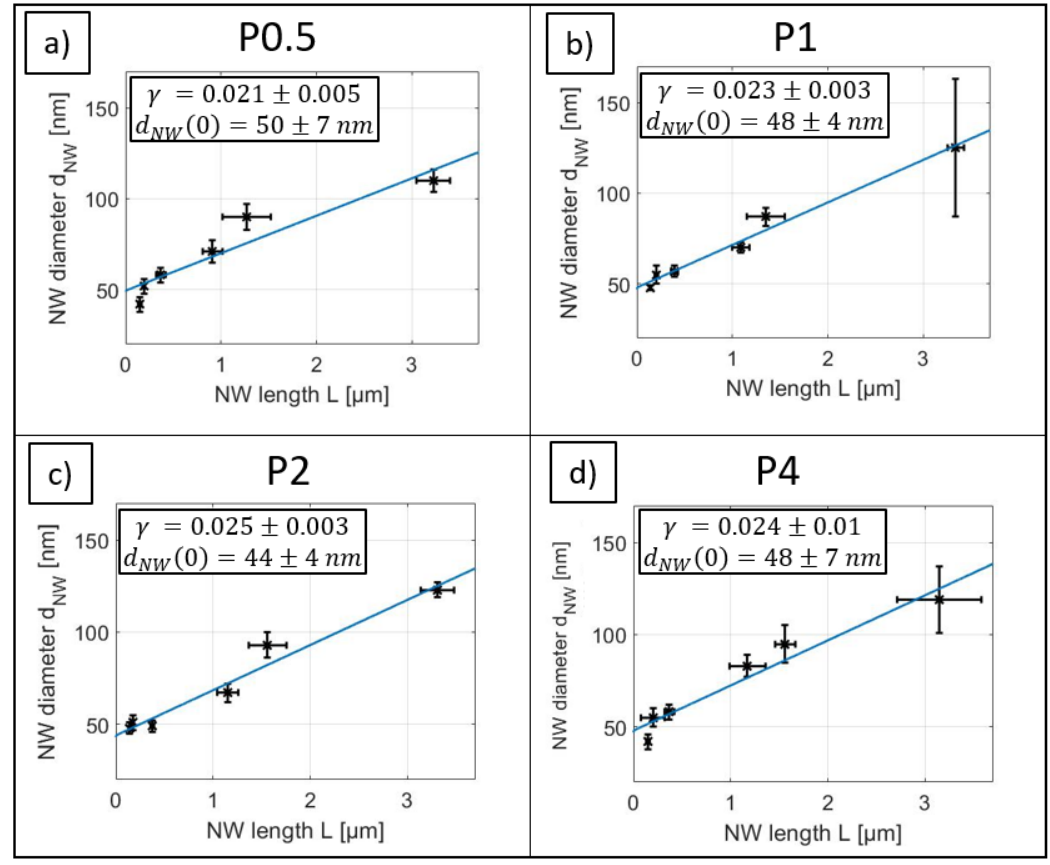

Figure S4. NW diameter as a function of NW length for subset $A$ (hole diameter of $80 \mathrm{~nm}$ ) including the different pitches ranging from 0.5 to $4 \mu \mathrm{m}(a)-d)$ ). The resulting anisotropy factors and initial NW diameters are determined by linear fits.

Subset B

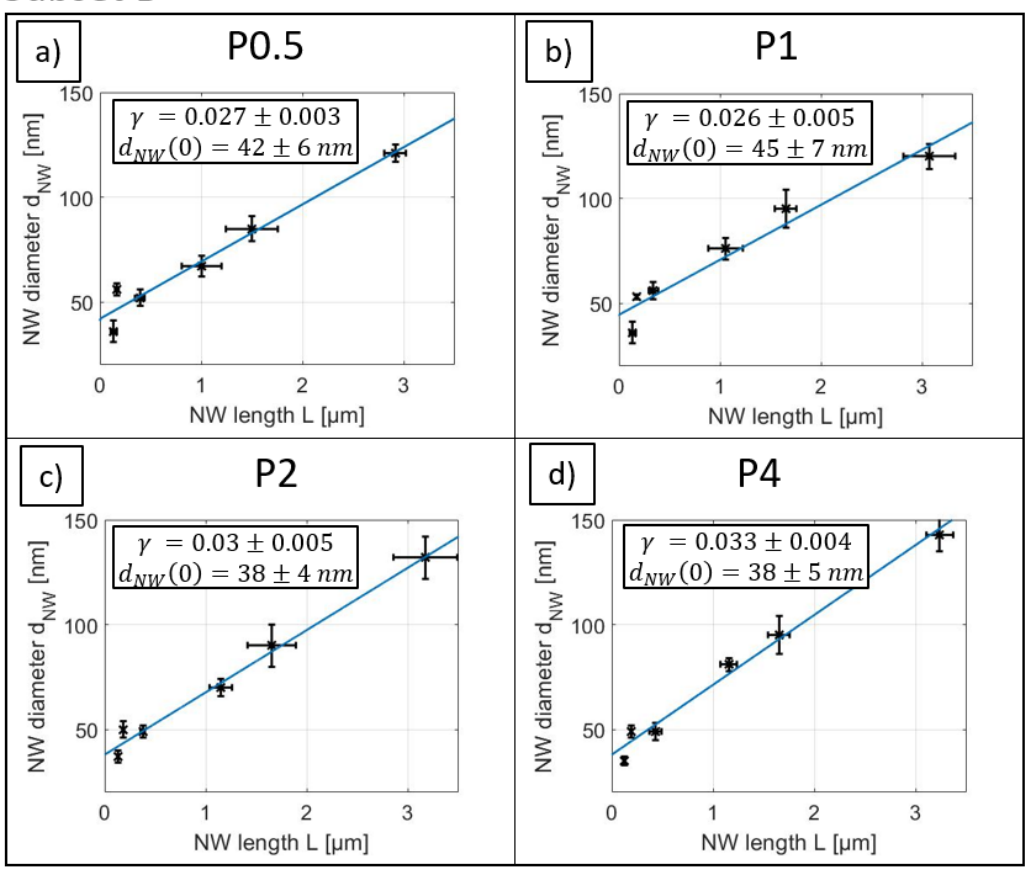

Figure S5. The NW diameter as a function of the NW length for subset B (hole diameter of $60 \mathrm{~nm}$ ) NWs and corresponding linear fits. 
For all pitches in subset A we observe only slight variations in the anisotropy factor and the initial NW diameter, which suggests independence of the NW growth dynamics from the pitch. In subset B (see Fig.S5), a slight increase of the anisotropy factor and a decrease of the initial NW radius is observed. These effects are attributed to the smaller hole diameter in subset $B$, which likely leads to a reduced initial NW diameter. However, we did not observe any additional pitch dependence on the NW growth. Finally, we analyzed NWs of subset C in Fig. S6 where the variations of the anisotropy factor and the initial diameter are substantially fortified. We attribute this to a truncated conical shape of the holes with the smallest diameter as discussed below in the analysis of the contact angles in all configurations.

Subset C

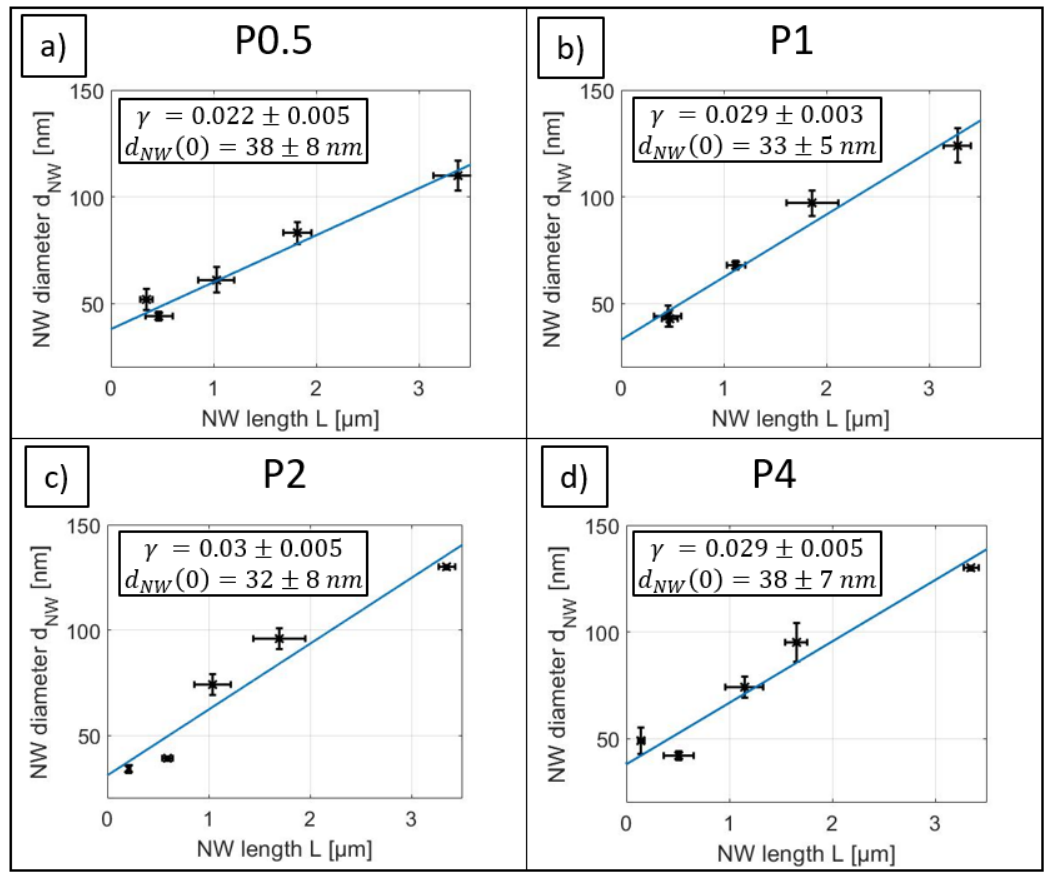

Figure S6. The NW diameter as a function of the NW length of subset C (hole diameter of $40 \mathrm{~nm}$ ) NWs and corresponding linear fits.

\section{Measurements of the contact angle}

The obtained contact angle data were calibrated by comparing SEM measurements of as grown GaAs NWs examined under $90^{\circ}$ (see Fig. S7a) and under $40^{\circ}$ tilt (see Fig.S7b), respectively. The former are performed on scattered NWs on a TEM grid, while the latter are taken from free-standing NWs on the

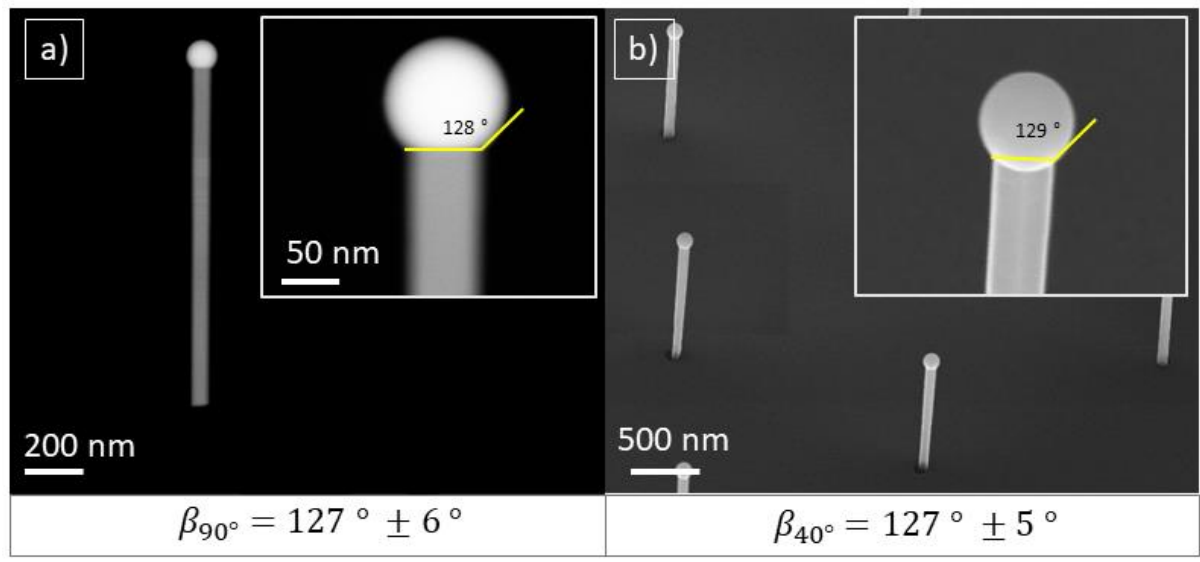

Figure S7. a) Measurement of the contact angle on an exemplary NW under $90^{\circ}$ angle on a TEM grid in the SEM. b) Contact angle estimated from standing NWs under $40^{\circ}$ angle in the SEM. 
substrate. Overall, $9 \mathrm{NWs}$ were analyzed in the $90^{\circ}$ setting, while more than $80 \mathrm{NWs}$ were investigated in the $40^{\circ}$ setting. The measurements are in good agreement and we conclude that the contact angle can be measured under $40^{\circ}$ tilt without substantial deviations from the measurements under $90^{\circ}$. This calibration was also performed for Ga droplets with contact angles around $90^{\circ}$ and a similar good agreement between both measurement techniques was achieved.

\section{Contact angle evolution}

The time evolution of the contact angle is depicted in Figs. S8 - S10 for all subsets. The continuous line is obtained from the simulation and the light blue area reflects the variation of the model results considering the standard deviations $\pm \sigma$ for the material parameters (Tab.1 in the paper). In Figure S8 the experimental data and the simulation of the contact angle for subset A NWs is presented. The simulation is in good agreement with the experiments and has a standard deviation of $3-10^{\circ}$. For all

Subset A

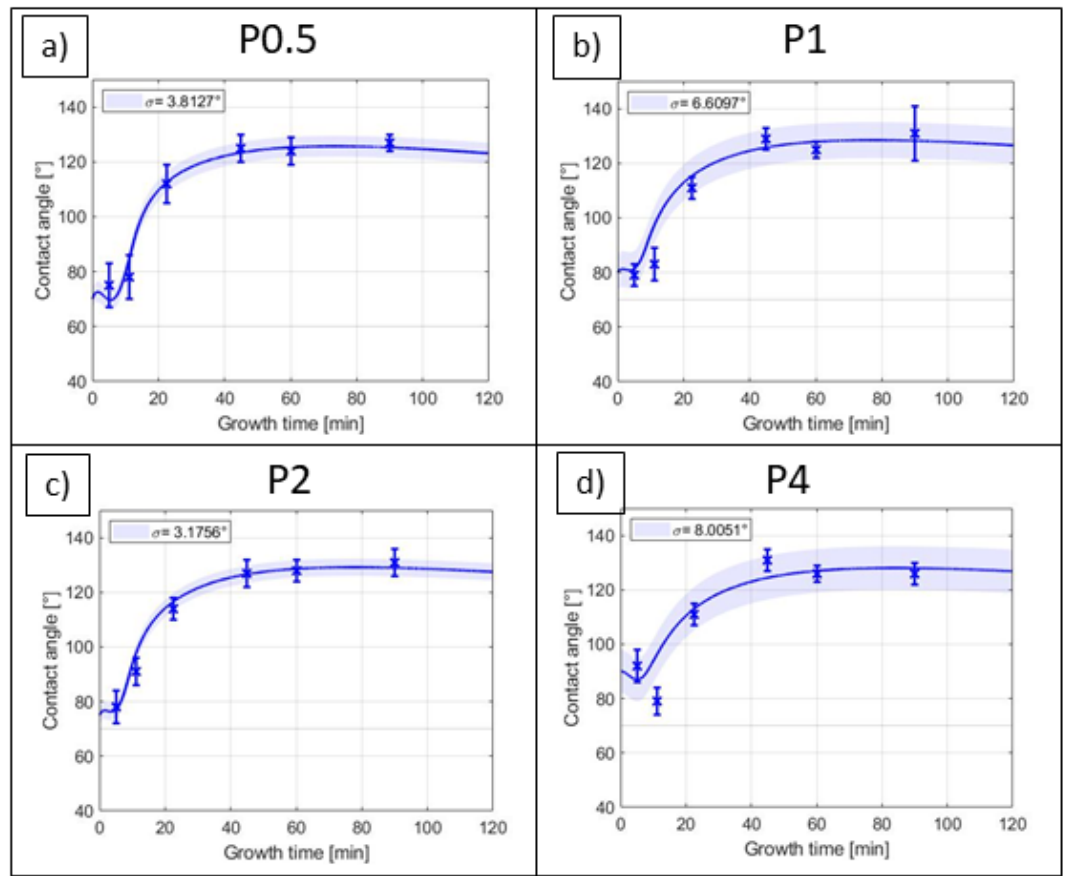

Figure S8. The Ga droplet contact angle evolution as a function of growth time for subset $A$ (hole diameter 80 of $\mathrm{nm}$ ) NWs. a)-d) are the four different pitches ranging from 0.5 to $4 \mu \mathrm{m}$. The light blue area represents the $\pm \sigma$ variation of the model calculation.

pitches, the best-fit calculation yields the same initial contact angle, which also confirms the independence of the NW growth start on the pitch. In Fig. S9, the contact angle evolution of subset B is shown. In this subset, the simulation is also in good agreement with the experiments showing standard deviations of $9-11^{\circ}$. Note that within the first $5-30$ min of NW growth the time evolution of the contact angle proposed by our model slightly differs between the two subsets $A$ and $B$. This is likely a result of small variations in the starting conditions (e.g. variations in $\mathrm{SiO}_{2}$ thickness and hole shape) 
and can be more pronounced for smaller hole diameters. Afterwards, the contact angle stabilizes to $\sim 125^{\circ}$. Overall, our studies indicate that for both subsets the initial contact angle varies between $\sim 70-90^{\circ}$.

\section{Subset B}

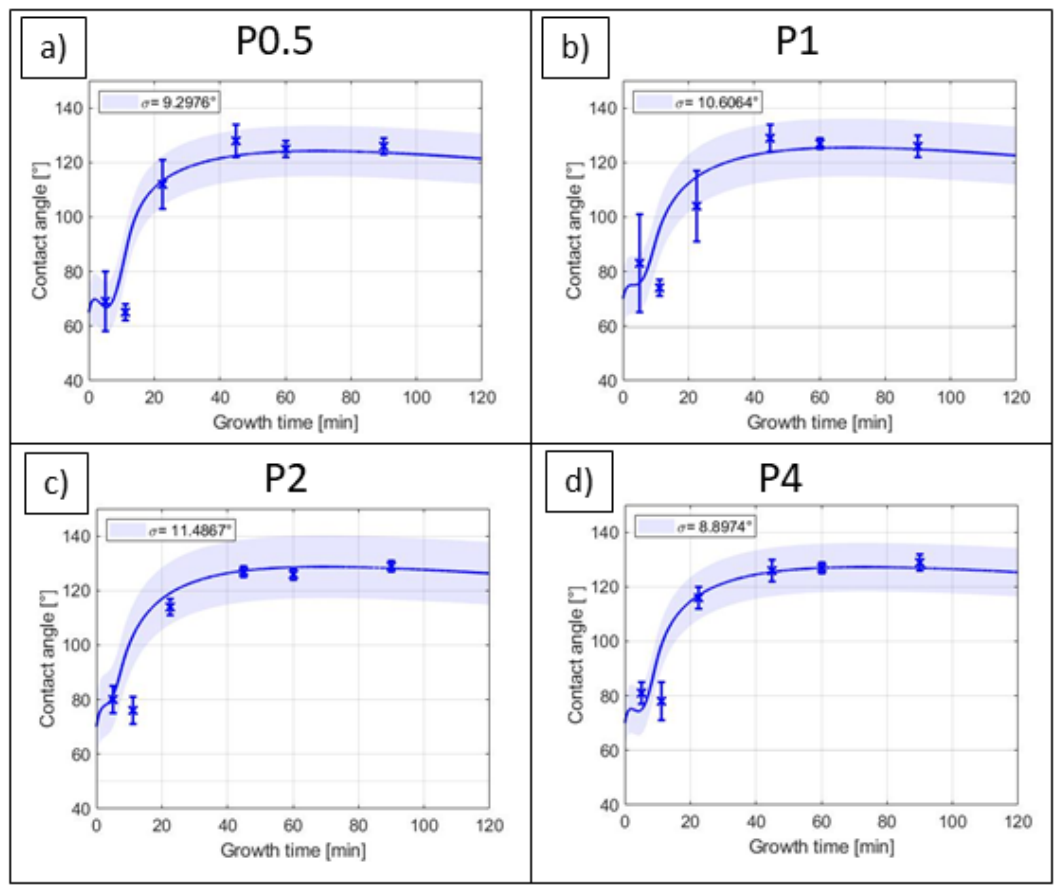

Figure S9. The Ga droplet contact angle evolution as a function of growth time for subset $B$ (hole diameter of $60 \mathrm{~nm}$ ).

However, we observe a substantial increase of the initial contact angles in subset C NWs shown in Fig. S11. We suspect that the final wet-chemical fabrication step of the PP substrates by HF leads to a truncated conical shape of the hole. ${ }^{s 3}$ For smaller diameters, this affects the hole geometry by

Subset C

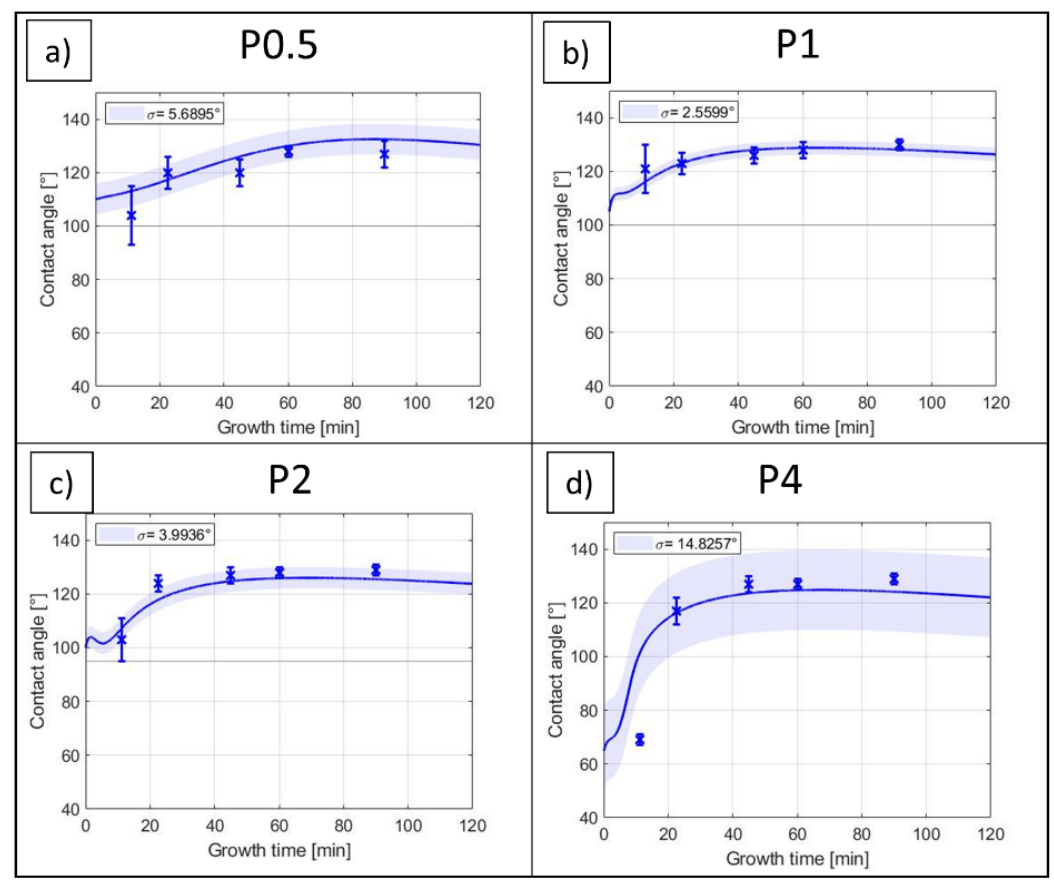

Figure S10. The Ga droplet contact angle evolution as a function of growth time for subset $C$ (hole diameter of $40 \mathrm{~nm}$ ). 
shrinking the area for the droplet formation and then potentially promotes larger contact angles at the beginning.

In case of NWs grown in subset A and B our studies indicate no influence of the pitch on the contact angle of the NW, neither for short nor long growth times. In accordance with our model this indicates that the diffusion length of $\mathrm{Ga}$ adatoms on the $\mathrm{SiO}_{2}$ surface is equal or less than $250 \mathrm{~nm}$, i.e. $50 \%$ of the smallest pitch $(500 \mathrm{~nm})$ provided by our PP substrates.

Additionally, we precisely adjusted our model to fit the measured NW length evolution for all configurations. The simulations are again divided into the three subsets $A, B$ and $C$ and presented in Figs. S11-S13. Again, 5 to 15 individual NWs were analyzed for each data point and the error bar corresponds to the standard deviation $\pm \sigma$ of the mean average value. Fig. S11 shows the length evolution for NWs of subset A. The NW length increases superlinear to about $3 \mu \mathrm{m}$ after $90 \mathrm{~min}$ growth. The superlinear time evolution of the NW length can be decomposed into two stages with different axial growth rates, the first stage ranging from 5-20 min and the second from 20-90 min. This two- stage NW length evolution was also observed in recent studies and results from the interplay of the main $\mathrm{Ga}$ diffusion pathways to the droplet (e.g., via $\mathrm{SiO}_{2}$ and via the side facets ${ }^{S 1}$ ). As before, the light blue area covers the standard deviation of our model calculations. The latter are $0.16-0.36$ $\mu \mathrm{m}$ and for all pitches, we obtained a good agreement with the experimental data. The length evolution of NWs in subset B and C are shown in Fig. S12 and Fig. S13. Corresponding standard deviations of the model calculations are $0.22-0.28 \mu \mathrm{m}$ and $0.35-0.40 \mu \mathrm{m}$, respectively.

\section{Subset A}

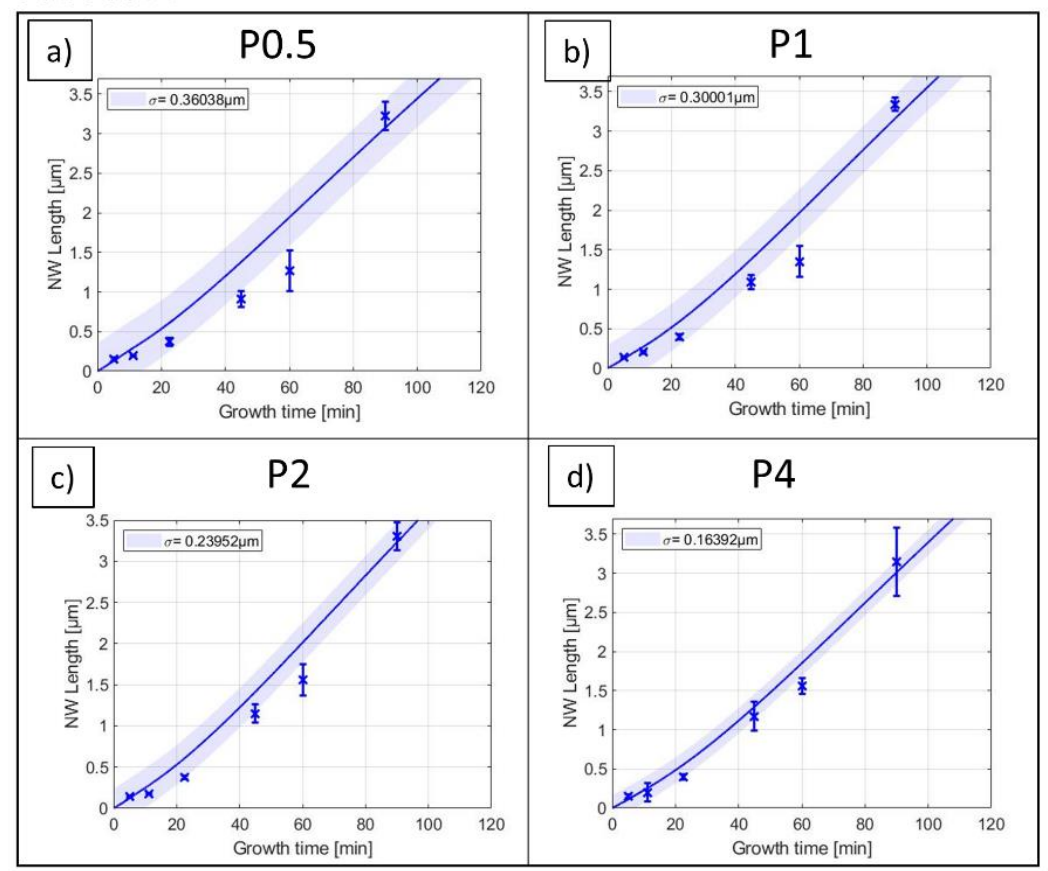

Figure S11. The NW length evolution as a function of growth time for subset $A$ (hole diameter of $80 \mathrm{~nm}$ ) NWs. a)-d) are the four different pitches ranging from 0.5 to $4 \mu \mathrm{m}$. The light blue area represents the $\pm \sigma$ variation of the model calculation. 


\section{Subset B}

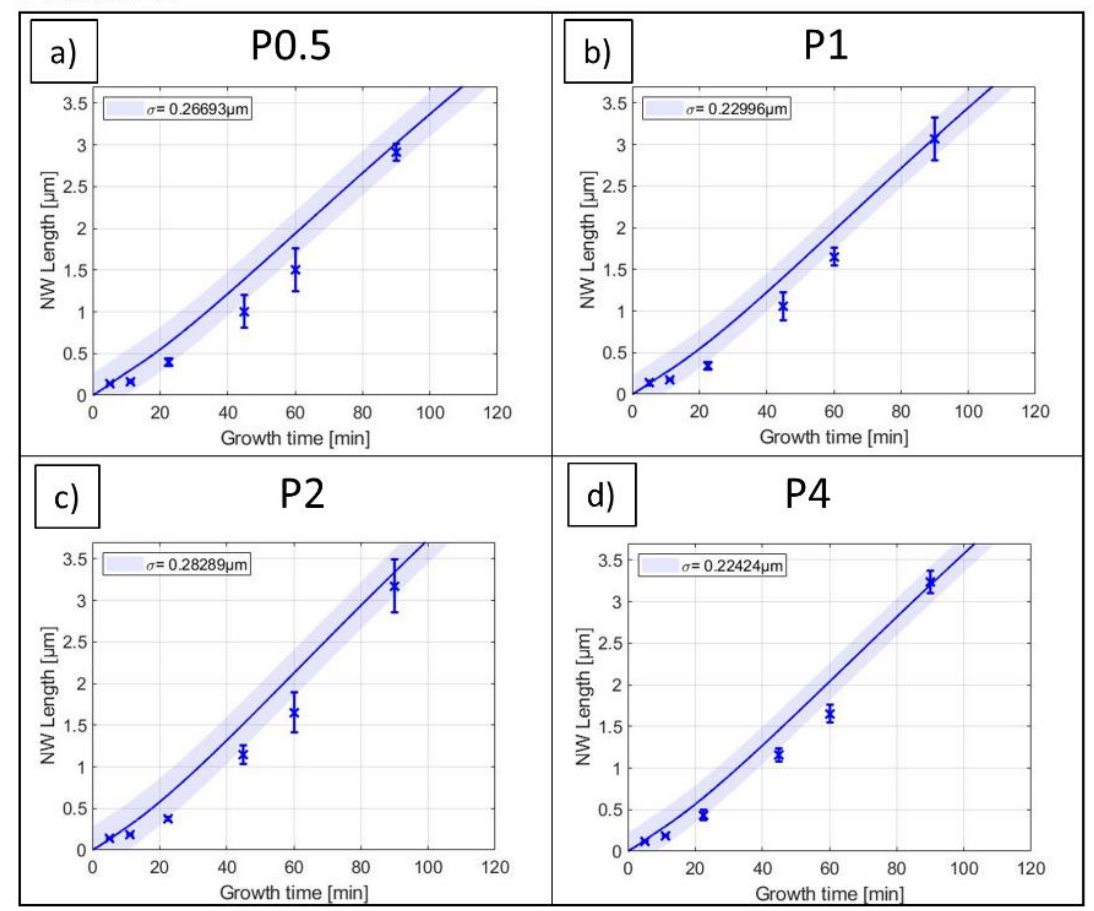

Figure S12. The length evolution as a function of growth time for subset B (hole diameter of $60 \mathrm{~nm}$ ) NWs.

\section{Subset C}

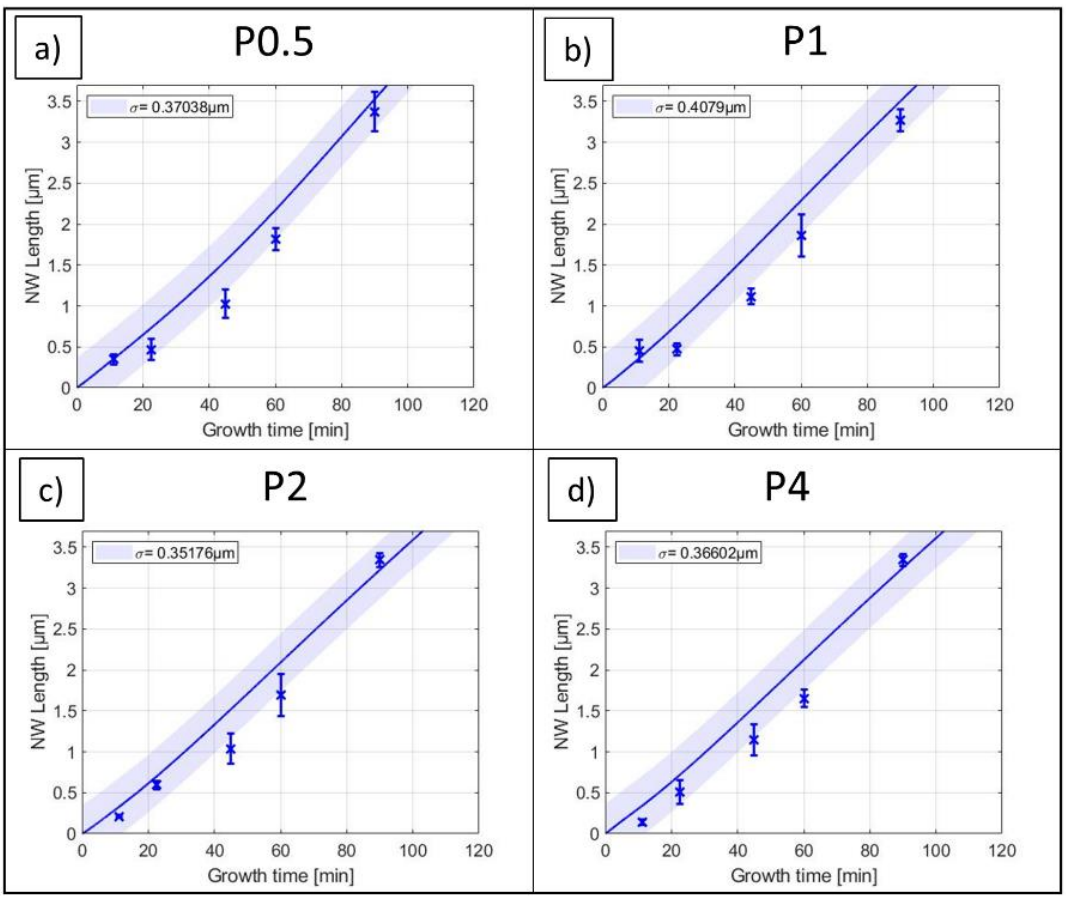

Figure S13. The length evolution as a function of growth time for subset $\mathrm{C}$ (hole diameter of $40 \mathrm{~nm}$ ) NWs.

Using the full set of data (Figs. S4 - S13) obtained from the growth dynamics of NWs grown with a constant Ga flux we refined our model to a small set of remaining parameters and calculated the dynamic Ga flux dependence presented in Fig. S14. The latter starts with a constant initial Ga flux of $100 \%$ for the first $10 \mathrm{~min}$, which is the same as used for the regular NW growth experiments. Afterwards, the Ga flux is reduced to approx. $60 \%$ of the initial Ga flux. This is experimentally implemented by multiple linear temperature ramps of the Ga cell. The light blue area covers the variation of the Ga flux when the standard deviations of the starting conditions $\left(r_{N W}(0), \gamma\right.$ and $\left.\beta(0)\right)$ 
are considered. Altogether these initial errors lead to a variation of about $\pm 10 \%$ in the Ga flux curve over the entire growth period. This is likely comparable to the variations of subsequent Ga BEP measurements at a constant cell temperature. Consequently, our calculated Ga flux curve provides sufficient accuracy to meet the requirements for stabilization of the contact angle to a certain target value.

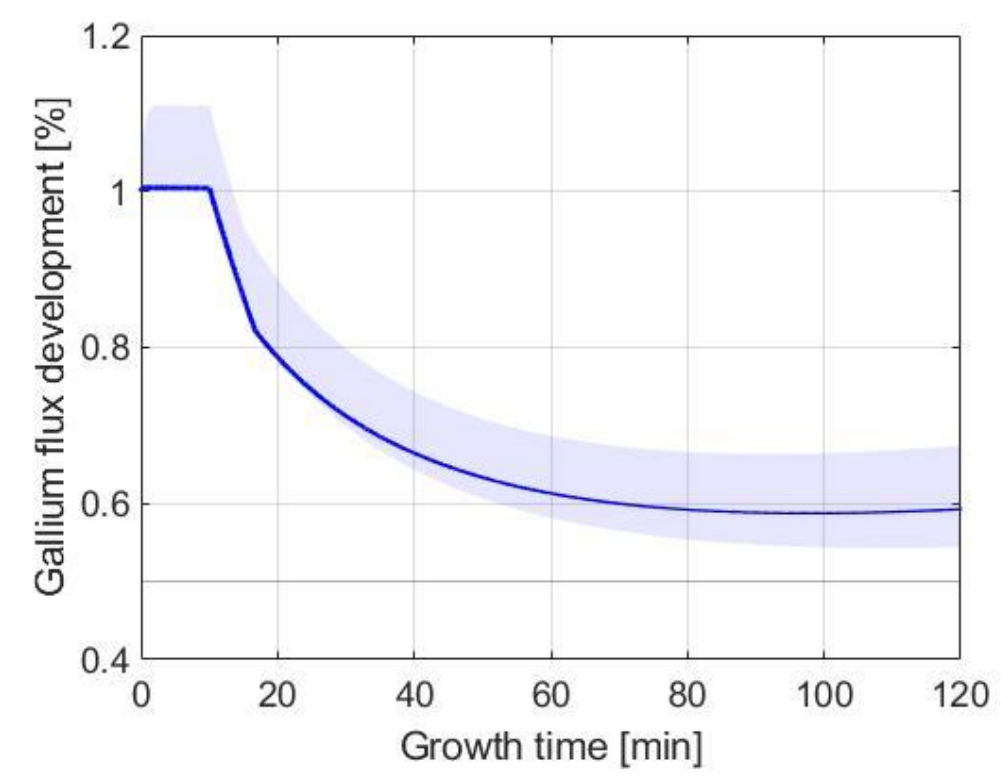

Figure S14. Calculated dynamic Ga flux curve used for our phase-pure growth experiment. The light blue area covers the interval of $\pm \sigma$ variation of $\gamma, r_{N W}(0)$ and $\beta(0)$.

\section{S3 Calculation and calibration of the material fluxes}

\section{Calculation of the incoming $\mathrm{Ga}$ and $\mathrm{As}_{4}$ fluxes}

The Ga and As flux is calculated using the Hertz-Knudsen equation:

$$
J_{\text {flux }}\left[\text { atoms } m^{-2} s^{-1}\right]=\frac{p}{\sqrt{2 \pi k_{B} T M}}=\frac{2.6358 \cdot 10^{24}}{0.0075} \cdot \frac{p[\text { torr }]}{\sqrt{T[K] \cdot M[u]}}
$$

with the effusion cell beam equivalent pressure (BEP) $p$, the cell temperature $T$, the atomic mass $M$ and the Boltzmann constant $k_{B}$. Since the sensitivity of the ion gauge is calibrated to nitrogen, a correction factor is needed to accurately describe the $G$ a and As fluxes. For that purpose, the growth rate of $\mathrm{Ga}$ is measured with high certainty using RHEED. By comparing the measured Ga flux (Ga BEP is inserted in the Hertz-Knudsen equation) with the theoretically necessary flux to obtain the measured growth rate, and by considering the respective geometry of the MBE system, we derived a calibration factor of $c_{f, G a}=3.8$. To calibrate the $A s_{4}$ BEP we used the calibration factor obtained for Ga and included the ionization efficiencies $\eta_{i}$ for both materials:

$$
c_{f, A s}=c_{f, G a} \cdot \frac{\eta_{G a}}{\eta_{A s}}
$$


The ionization efficiencies are given relative to nitrogen by $\frac{\eta_{i}}{\eta_{N_{2}}}=0.4 \cdot \frac{Z_{i}}{14}+0.6$ and with that, the calibration factor for $A s_{4}$ is $c_{f, A s}=1.3$.

\section{Calculation of the surface area of the NW side facets}

The rotation of the substrate holder must be considered to calculate a time-average flux impinging onto the side facets of a single NW. The rotation was set to $10 \mathrm{rpm}$, which results in an angle variation of $60^{\circ}$ per second. In addition to the unshadowed half $\left(180^{\circ}\right)$ of the NW exposed to the flux without rotation, the latter increases the effective surface area to $240^{\circ}$ within a one-second time step, which leads to a correction factor of $2 / 3$.

\section{Direct impingement collection area of the Ga droplet}

To derive the surface area of the Ga droplet, we calculate the surface area of a quarter of a circle by:

$$
A=\int_{0}^{y} r_{i} d h
$$

where $r_{i}$ is given by the Pythagorean theorem $r_{d r o p l e t}^{2}=r_{i}^{2}+y^{2}$. Then, the integration gives

$$
A(\beta, r)=\int_{0}^{y} \sqrt{r_{\text {droplet }}^{2}+h^{2}} d h=\frac{r^{2} \arcsin \left(y / r_{\text {droplet }}\right)}{2}+\frac{y \sqrt{r_{\text {droplet }}^{2}-y^{2}}}{2}
$$

When the quarter of a circle is complete (e.g., $\left.y=r_{\text {droplet }}\right)$, the surface area is given by $A=$ $\pi \cdot r_{\text {droplet }}^{2} / 4$. When the quarter is incomplete $y$ is given by the contact angle and is described by $y(\beta)=r_{\text {droplet }} / \sqrt{1+\tan ^{2} \beta}$.

The area of the droplet surface facing the beam parallel to the substrate surface is given by

$$
A_{\|}(\beta, r)=\frac{\pi \cdot r_{d r o p l e t}^{2}}{2}+2 \cdot A(\beta, r)
$$

The droplet relevant surface area orthogonal to the substrate is set by

$$
A_{\perp}(r)=\pi \cdot r_{\text {droplet }}^{2}
$$


We have grown three samples (defined as type-2 NWs in the paper) using the adjusted Ga flux and varied the growth time (i.e., $30 \mathrm{~min}, 60 \mathrm{~min}$ and $90 \mathrm{~min}$ ). The representative HR-TEM analysis of one exemplary NW (after 90 min of NW growth) is shown in Fig. 6 of the text. However, we analyzed in total $16 \mathrm{NWs}$ including all growth experiments. In the following, HR-TEM studies of exemplary NWs obtained after $30 \mathrm{~min}$ and $60 \mathrm{~min}$ growth are presented.

\section{$30 \min$}

The crystal phase analysis of one exemplary type-2 NW grown for $30 \mathrm{~min}$ is displayed in Fig. S15. The NW was examined by HR-TEM measurements on the top (A), middle (B) and bottom (C) sections, it is $505 \mathrm{~nm}$ long and $100 \%$ phase-pure WZ. The absence of phase changes in this NW confirms the stability of the contact angle within the WZ growth regime.

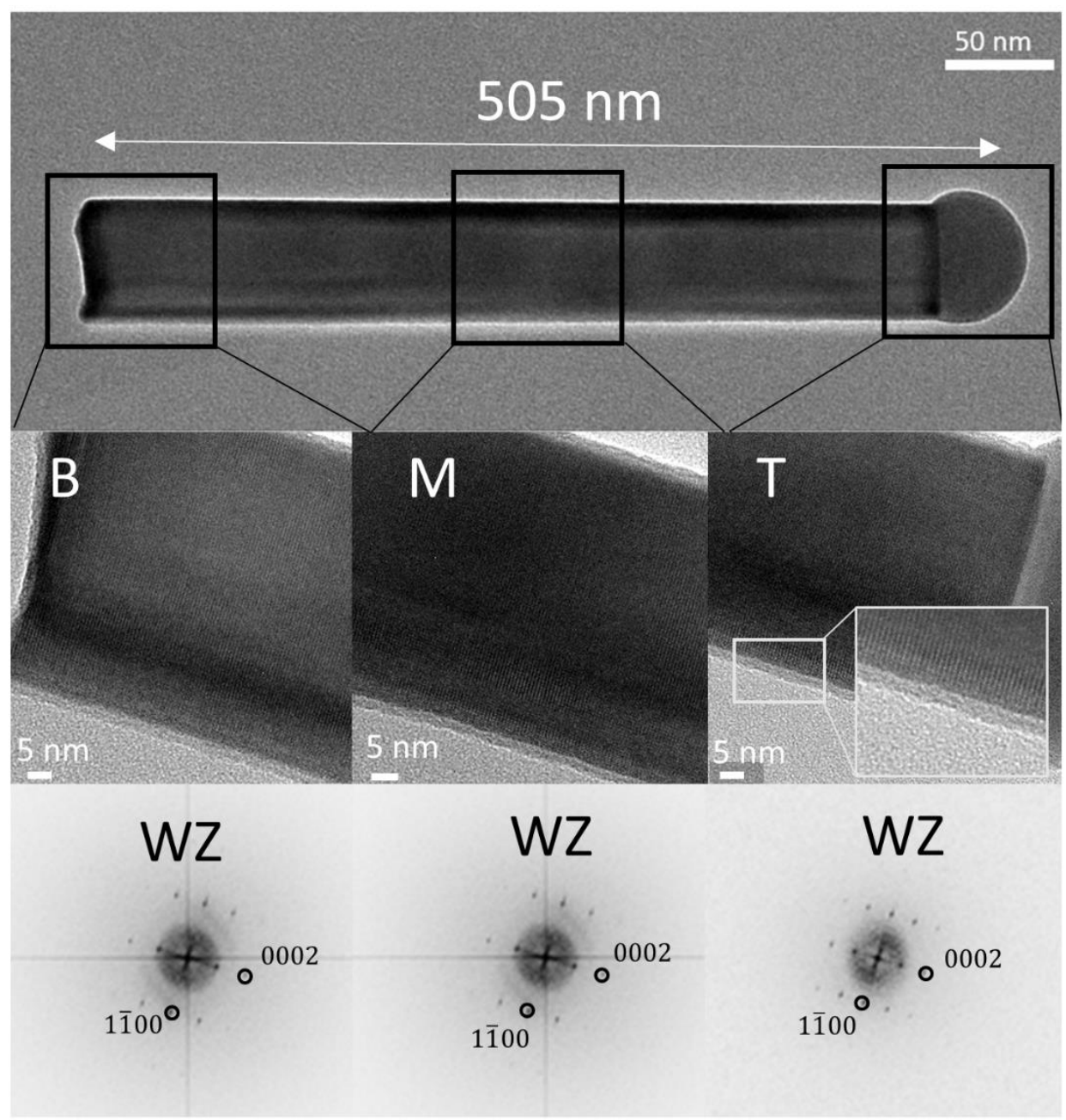

Figure S15. HR-TEM studies (in [110] for ZB or [112̄0] for WZ zone axis) of a typical GaAs NW after 30 min growth time. (T) Phase-pure WZ at the top of the NW with a HR-TEM insert highlighting the fringes of the NW. The middle (M) and bottom (B) sections are also analyzed by HR-TEM. The crystal phase is WZ along the entire NW. 


\section{$60 \mathrm{~min}$}

The crystal structure along one exemplary GaAs NWs grown for $60 \mathrm{~min}$ is shown in Fig. S16. It is 870 $\mathrm{nm}$ long and consists of $99.9 \%$ WZ crystal phase with five stacking faults. The Ga droplet contact angle of about $113^{\circ}$ is in the WZ phase growth regime. ${ }^{s 4}$

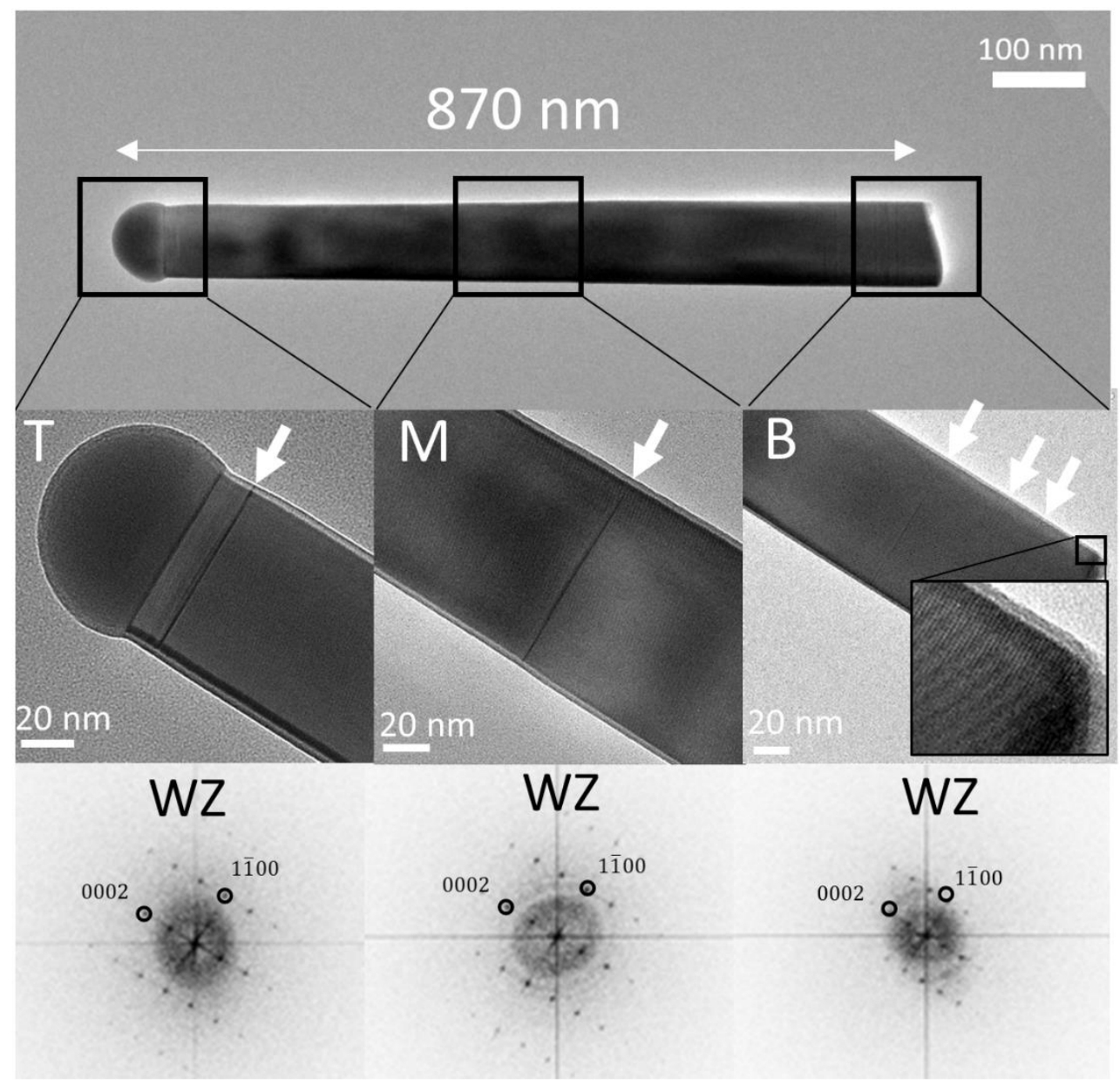

Figure S16. HR-TEM studies (in [110] for ZB or [112̄0] for WZ zone axis) of an exemplary GaAs NW after 60 min growth time. (T) Phase-pure WZ at the top of the NW. The middle (M) and bottom $(B)$ section, with a HR-TEM insert highlighting the fringes of the NW, contain several ZB inclusions.

The stacking faults are indicated by white arrows in Fig. S16. The bottom WZ section contains three of them, while the grade of phase-purity is substantially higher in the middle (see Fig. S16 (M)) and top (T) section (see Fig. S16) with only one stacking fault in each segment.

Figure S17 shows HR-TEM images taken from another type-2 NW grown for $60 \mathrm{~min}$. The length of this NW is about $1.75 \mu \mathrm{m}$ and the HR-TEM analysis (see S17 T, M and B) reveals $100 \%$ phase-pure ZB crystal structure and not any twin boundary all along the entire NW. Unlike the above-mentioned NW shown in Fig. S17, the initial contact angle for this NW was likely higher than expected, which promotes the growth of ZB crystal structure $\left(\beta>125^{\circ}\right)$.

Initial contact angles in this range were observed for NWs grown in the holes with the smallest diameter (subset C). Interestingly, the Ga droplet contact angle on top of this NW is about $112^{\circ}$, which is in the WZ-stabilized growth regime. However, the occurrence of the ZB crystal phase in this regime can be explained by the hysteresis of the contact angle transition (i.e., from higher to lower contact 


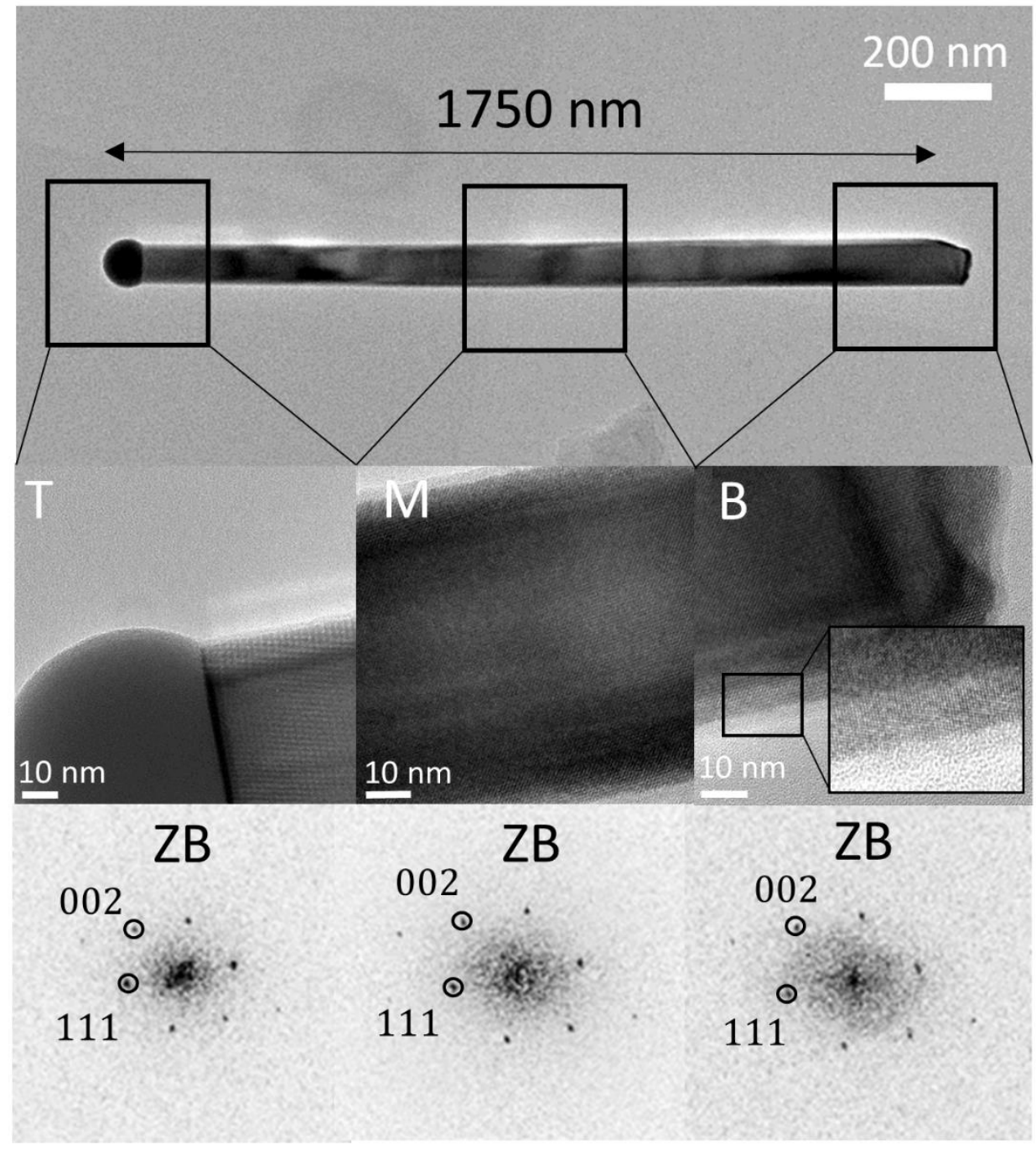

Figure S17. HR-TEM studies (in [110] zone axis) of an exemplary GaAs NW after 60 min growth time. (A) Phase-pure ZB at the top of the NW. The middle (B) and bottom (C) section, with a HRTEM insert highlighting the fringes of the NW, are also examined by HR-TEM analysis and reveal a phase-pure ZB NW without any WZ inclusions.

angles values or vice versa) observed by Panciera et al.. ${ }^{55}$ They observed ZB growth for Ga droplet contact angle in the range of $110^{\circ}$, when the contact angle decreases from a higher value (around $130^{\circ}$ ) down to $110^{\circ}$. Note, that increased length and reduced diameter of these ZB NWs (compared to the WZ NWs), is expected due to the small hole diameter in subset C. This has two effects: Firstly, the initial droplet size is smaller, which leads to a reduced initial NW diameter. Secondly, the contact angle is larger which leads to an increased droplet surface area. The latter induces an enhancement of the direct impingement rate of As atoms on the droplet surface, and with that a higher growth rate (see also Eq. 6 in the manuscript). 


\section{S5 Supplementary references}

S1. Vettori, M., Danescu, A., Guan, X., Regreny, P., Penuelas, J. \& Gendry, M. Impact of the Ga flux incidence angle on the growth kinetics of self-assisted GaAs nanowires on $\mathrm{Si}(111)$. Nanoscale Adv. (2019) doi:10.1039/c9na00443b.

S2. Rieger, T., Lepsa, M. I., Schapers, T. \& Grützmacher, D. Controlled wurtzite inclusions in selfcatalyzed zinc blende III-V semiconductor nanowires. J. Cryst. Growth 378, 506-510 (2013).

S3. Vettori, M., Piazza, V., Cattoni, A., Scaccabarozzi, A., Patriarche, G., Regreny, P., Chauvin, N., Botella, C., Grenet, C., Penuelas, J., Fave, A., Tchernycheva, M. \& Gendry, M. Growth optimization and characterization of regular arrays of GaAs/AlGaAs core/shell nanowires for tandem solar cells on silicon. Nanotechnology 30, (2019).

S4. Dursap, T., Vettori, M., Danescu, A., Botella, C., Regreny, P., Patriarche, G., Gendry, M. \& Penuelas, J. Crystal phase engineering of self-catalyzed GaAs nanowires using a RHEED diagram. Nanoscale Adv. 2127-2134 (2020) doi:10.1039/d0na00273a.

S5. Panciera, F., Baraissov, Z., Patriarche, G., Dubrovskii, V. G., Glas, F., Travers, L., Mirsaidov, U. \& Harmand, J. C. Phase Selection in Self-catalyzed GaAs Nanowires. Nano Lett. 20, 1669-1675 (2020). 